\title{
DIFICULTADES EPISTEMOLÓGICAS Y TÉCNICAS EN ENCUESTAS A POBLACIÓN ELUSIVA: EL CASO DE LOS TURISTAS RESIDENCIALES
}

\author{
José Andrés Domínguez \\ Universidad de Huelva \\ Antonio Aledo ${ }^{2}$ \\ Universidad de Alicante \\ Bernat Roig Merino ${ }^{3}$ \\ Universitat Politècnica de València
}

\begin{abstract}
RESUMEN
Este artículo muestra una variedad de posibles soluciones ante las dificultades que los científicos sociales pueden encontrarse en la investigación cuantitativa con poblaciones elusivas. Estas dificultades son de dos tipos: epistemológicas y técnicas. Desde el punto de vista epistemológico, se subraya la necesidad de adaptación de la investigación a las características de la población objeto de estudio, pues la elusividad de la población no puede considerarse un obstáculo insalvable. Esta flexibilidad se muestra más constructiva, en términos de consecución de objetivos de investigación, que un posicionamiento más rígido o tradicional, más apegado a modelos preestablecidos. Desde el punto de vista técnico, se muestran las soluciones ante cada dificultad encontrada en el curso de un estudio de caso: la población turístico-residencial del destino Costa Blanca en el Levante español. Estas soluciones se demuestran, finalmente, como adecuadas al contrastar los resultados con encuestas oficiales tipo panel realizadas de forma tradicional en origen.
\end{abstract}

Palabras clave: demanda turística; flexibilidad metodológica; métodos cuantitativos; poblaciones elusivas; veraneantes.

Recibido: 28 de mayo de 2014

Devuelto para su revisión: 7 de noviembre de 2014

Aceptado: 19 de enero de 2015

1 Departamento de Sociología y Trabajo Social. Universidad de Huelva. Campus El Carmen. 21071 HUELVA (España).E-mail: andres@uhu.es

2 Departamento de Sociología I. Universidad de Alicante. Edif. Ciencias Sociales. Campus San Vicente del Raspeig. 03690.ALICANTE (España).E-mail: antonio.aledo@ua.es

3 Departamento de Economía y Ciencias Sociales. Universitat Politècnica de València . C/ Paranimf $\mathrm{n}^{\circ} 1$. 46730 Grao de Gandía .VALENCIA (España). E-mail: bernatrm@esp.upv.es 


\title{
Epistemological and technical difficulties in surveys for elusive population: the case of residential tourism
}

\begin{abstract}
Social researchers can find many difficulties dealing with elusive populations, and this article shows epistemological and technical solutions for this cases. From an epistemological point of view, it is necessary a strategic adaptation to the population features, since methodological problems derived from elusive condition of population cannot be considered as insurmountable obstacles. That skill (methodological adaptation or flexibility) proves its potential for achieving research aims, far away from more rigid or «traditional» proposals (bonding with pre-established models). From a technical point of view, specific solutions for several arisen difficulties are shown, using the case of anon-site survey to residential summer tourists at the Costa Blanca, a mature Spanish destination. These solutions are finally validated by contrasting their results with the results of an official household panel survey, produced on a traditional way, that is, getting data from tourists whilst they are at their homes after their summer holidays.
\end{abstract}

Keywords: elusive population; methodological flexibility; quantitative methods; tourism demand; residential tourism.

\section{INTRODUCCIÓN}

A pesar de que los españoles optan mayoritariamente para sus vacaciones de verano por el alojamiento extra-hotelero, ya sea en su segunda residencia, vivienda de familiares y amigos o en alquiler, son pocos los estudios que se han acercado a este fenómeno de forma cuantitativa, especialmente tomando como área de estudio los destinos turísticos escogidos por este segmento turístico. Este déficit es significativo, pues son millones los turistas residentes en España los que conforman este grupo. En 2010, el 73,3\% de los veraneantes empleaban estas fórmulas de alojamiento durante sus viajes turísticos dentro de España (FAMILITUR, 2008). Su importancia no es solo numérica. Los efectos del desarrollo urbanístico asociado al turismo residencial han cambiado radicalmente decenas de poblaciones costeras (Vera, 1997), tanto en términos morfológicos como de estructura económica. Ello ha dado lugar a una dependencia casi total de estos municipios respecto del sector inmobiliario que satisface las demandas de viviendas secundarias de estos veraneantes. Un mejor conocimiento de este segmento redundaría en una mejor gestión turística a escala municipal.

Es posible explicar este déficit en los estudios turísticos españoles haciendo referencia a tres argumentos. Por un lado, se ha mencionado que desde una parte de la Academia se da una mirada despreciativa sobre el fenómeno del veraneo.

El veraneo se ha asociado a un turismo masificado, banal e incluso alienador para la clase trabajadora (Obrador, Crang y Travlou, 2009). En segundo lugar, se percibe un cierto desinterés en la promoción de investigaciones sobre un sector, el de la promoción turístico-residencial, que ha estado ligado a fenómenos de corrupción urbanística (Naredo 
y Montiel, 2011). Por último, las dificultades metodológicas que conlleva el estudio de los veraneantes en destino dificultan también la producción de información y conocimiento. Sin duda, a estas dificultades metodológicas contribuyen los problemas surgidos en la definición y delimitación teórica del fenómeno turístico-residencial (Huete y Mantecón, 2011). El propio concepto turismo residencial pretende englobar una realidad compleja en la que las actividades turística, urbanística y migratoria se hallan estrechamente unidas. Este término hace referencia a la demanda turística, pero también a la oferta de vivienda y de otros servicios, derivada tanto de los usuarios de segunda residencia con motivos fundamentalmente vacacionales como de las migraciones internacionales de retirados (García-Andreu, 2005). Este artículo se centra principalmente en este tercer grupo de dificultades referidas al ámbito metodológico y técnico.

Las dificultades y déficits señalados no deberían ser impedimentos para el estudio de las características y hábitos de los turistas veraneantes residenciales en España. A la importancia numérica y socioeconómica del turismo residencial (Aledo, 2008; EXCELTUR, 2005) se unen, como justificación de la necesidad de su análisis, los fuertes impactos del fenómeno en términos ambientales (Almenar, Bono y García, 2000; OSE, 2006), demográficos (Casado-Diaz, 1999; Rodríguez e Warnes, 2002), socioculturales (Torres, 2003:65) y políticos (Fernández, 2006; Roger, 2007). Es necesario conocer el proceso de producción del turismo residencial y su demanda para establecer estrategias de desarrollo local a corto, medio y largo plazo. Si bien, a nivel científico, su estudio implica un reto metodológico importante - dada, entre otros factores la elevada movilidad de los turistas y la falta de datos oficiales, especialmente a escala local-, su superación procuraría resultados muy útiles para las comunidades implicadas. No debe olvidarse que el turismo residencial es, para numerosos municipios del Mediterráneo español, el principal y casi único sector económico de referencia. Así, este artículo pretende, como objetivo central, mostrar las principales dificultades a las que se enfrentan los investigadores que orienten su trabajo hacia el estudio cuantitativo de los turistas residenciales. Se describe, en primer lugar, los retos afrontados en el ámbito epistemológico, relacionados con la naturaleza elusiva de la población objeto. En segundo lugar se ofrece, como orientación para casos similares, las soluciones adoptadas en un estudio específico, cuya contrastación de resultados con un estudio clásico de turistas en origen pone a prueba la validez de dichas soluciones. El estudio empleado como ejemplo consiste en una encuesta realizada a turistas veraneantes residenciales llevada a cabo en una muestra de municipios de la Costa Blanca (Alicante).

\section{ASPECTOS EPISTEMOLÓGICOS: ADAPTACIÓN AL OBJETO COMO CLAVE DE SU CONOCIMIENTO}

Para las ciencias sociales en general, y la sociología en particular, los problemas de medición del objeto de estudio son una constante desde su origen. No obstante, estas dificultades no son exclusivas de las ciencias sociales, pues también aparecen en las ciencias naturales, «clásicas», positivistas. El debate sobre las posibilidades de medición en las ciencias sociales recorre la tradición filosófica y sociológica (Cooper, King y Rettie, 2009; Durkheim, 1973; Hamel, 1992; Ho, 2008; Karlsen, Overland y Karlsen 2010; Kukla, 2000; Seiyama, 2006). Una de sus conclusiones más interesantes es que la obsesión por 
parecer científicos puede conllevar que elijamos nuestros objetos de estudio en función de si pueden o no ser investigados mediante metodologías cuantitativas, dado que estas metodologías son las de mayor reconocimiento e institucionalización científica.

Las ciencias sociales pueden asemejarse a veces a las ciencias «duras», pero en otros casos ni objeto ni métodos clásicos se ajustan a los objetivos del trabajo. El objeto requiere de ajustes epistemológicos y metodológicos, que a menudo se intentan evitar utilizando una rigidez impropia de sus necesidades, y que pueden incluso alterar la idea original de investigación al chocar contra los recelos de no saber cómo abordar el tema con las herramientas (clásicas, duras) de que disponemos. Diseñar un proyecto de investigación supone un ejercicio de planificación, y toda planificación conlleva un proceso de selección-descarte así como un ejercicio de gestión de la incertidumbre. La incertidumbre se debe manejar desde el método científico, empleando los conocimientos y herramientas que han producido las diferentes estrategias de investigación. Pero también juega un papel importante el «arte de investigar», es decir, la capacidad e incluso sensibilidad del investigador para enfrentarse a los imponderables de la investigación.

Así, la flexibilidad y adaptación al objeto por parte de la metodología se hace imprescindible si queremos realmente alcanzar nuestros objetivos de investigación (Díaz de Rada, 2006). Podemos desarrollar habilidades que cubran las particularidades del objeto con la utilización de métodos mixtos (Creswell y Plano, 2011:27; Harrison y Reilly, 2011:8), con la cualificación de los recursos humanos empleados en el proceso productivo de los datos y con estrategias de triangulación y muestreos no probabilísticos en busca de la confiabilidad suficiente para la toma de decisiones en la investigación (Ben-Haim, Zacksenhous, Keren y Dcaso, 2009; Fernández, 2003; Mainar y Grilló, 2006).

La necesidad de flexibilidad - entendida en este caso como la imprescindible adaptación metodológica a las características de la población que queremos conocer científicamente - es especialmente trascendente en el caso de las poblaciones denominadas elusivas (Sudman, Sirken y Cowan, 1988). El diccionario de la Real Academia Española define elusiva como esquiva, difícil de atrapar. Se trata, desde las ciencias sociales, de poblaciones sobre las que no existen registros oficiales, de las que no se conocen exactamente cuántos son ni dónde están. Son poblaciones caracterizadas por una intensa movilidad territorial y/o temporal o por situarse en intersticios del espacio social de difícil acceso. Algunos ejemplos de este tipo de población podrían ser los inmigrantes ilegales (Heckmann, 2004), las poblaciones afectadas por desastres ambientales (Henderson, Sirois, Chia-Chen, Airriess, Swanson y Banks, 2009), las personas sin hogar (David y Snijders, 2002) o, como en el caso objeto de este trabajo, la población turista veraneante que se aloja en unidades residenciales (Sharma, Dyer, Carter y Gursoy, 2008).

Se dan diversas razones para definir la población veraneante residencial como elusiva o, lo que es lo mismo, surgen diversas dificultades para conocer sus características y ubicación específica necesarias para el control técnico durante el proceso de muestreo convencional; a saber: 1) su dispersión territorial en destino, generado por el modelo de urbanismo extensivo que obstaculiza la localización de las viviendas ocupadas por los turistas residenciales; 2) su intensa movilidad y la dificultad para conocer los tiempos de estancia, dado que al ocupar las viviendas por razones de ocio gran parte de su tiempo lo disfrutan fuera de sus viviendas; y 3) la notable falta de datos oficiales sobre este colectivo 
a nivel municipal, ya que la ocupación de estas viviendas a lo largo del año no se recoge en los censos y padrones. A continuación se amplía este último punto que es especialmente relevante en términos de representatividad de la muestra en una investigación cuantitativa.

En las estadísticas oficiales del Instituto Nacional de Estadística (INE) sobre la oferta de alojamientos turísticos en España ${ }^{1}$ solo se lleva registro de los turistas que se alojan en hoteles, campings, casas rurales y apartamentos turísticos ${ }^{2}$. Por ello, la gran mayoría de los turistas que optan por un alojamiento residencial no son registrados oficialmente. No se hace un seguimiento a nivel municipal sobre el uso de este tipo de alojamientos. Y tampoco el Censo Nacional de Vivienda ofrece una información útil a este respecto. El INE realiza dicho censo cada diez años, pero solo distingue entre viviendas principales, viviendas secundarias y viviendas vacías. Esta información es insuficiente para conocer el número y características sociodemográficas de los usuarios de las viviendas en destinos turísticos ${ }^{3}$.

La única fuente oficial de datos de la que se dispone para la población veraneante nacional es la encuesta FAMILITUR (Encuesta de movimientos turísticos de los españoles). Se trata de una encuesta-panel realizada en hogares por el Instituto de Estudios Turísticos (IET) para conocer el comportamiento turístico de los residentes en España. Esta encuesta ofrece datos relevantes sobre el colectivo turístico-residencial, desagregados hasta la escala autonómica pero, a nivel municipal, los tamaños de muestra son demasiado reducidos para considerarlos fiables.

La escasez de datos oficiales, que dificulta el conocimiento de este segmento de la demanda turística, puede ser debida a razones de carácter socio-político. García-Andreu (2008) y Ortiz (2009) han demostrado que, en destinos como la Costa Blanca, el déficit de información está relacionado con la gestión política del urbanismo. La expansión del turismo residencial en dicho destino se ha caracterizado por su rápido crecimiento, escasa planificación y por una notable opacidad en los procesos de toma de decisiones relacionadas con el desarrollo urbanístico. Muy probablemente, los numerosos desmanes urbanísticos asociados al turismo residencial ayuden a comprender la falta de estudios previos y de datos fiables en destino sobre este tipo de turismo. Una gran parte de los actores clave dentro del sector turístico-residencial (propietarios, inquilinos, políticos locales, promotores...) son los primeros interesados en que no existan datos fiables sobre esta actividad económica.

En definitiva, resulta una tarea difícil y compleja conocer el universo de los turistas residenciales veraneantes en destino. No existen datos a escala municipal, ni sobre cuántos son, ni sobre la composición del grupo de viaje, ni sobre el uso temporal que hacen de

1 El Instituto Nacional de Estadística desarrolla la Encuesta de Ocupación en Alojamientos Turísticos.

2 Se considera apartamento turístico aquel que sale al mercado a través de canales oficiales y que computa impuestos. Estos apartamentos turísticos reglados constituyen una minoría frente a aquellas segundas residencias que salen al mercado de alquiler sin control de las administraciones turísticas.

3 El INE inició un proceso de normalización de los registros del Padrón Municipal de Habitantes y del Catastro, para poder cruzar más adelante ambas bases de datos. Estos trabajos, aunque costosos, habrían permitido en el medio plazo poder contar con un marco actualizado de Viviendas No Principales que podría haber sido utilizado en el futuro no solo para cuantificar la oferta del alojamiento privado de potencial uso turístico, sino también, mediante encuestas dirigidas a los propietarios, para saber quién lo utiliza, cuándo, cuánto tiempo y por qué (Mayo y Velasco, 2007). Este proyecto se ha paralizado por causas presupuestarias. 
sus viviendas orientadas al ocio residencial, ni sobre dónde se localizan. Y por tanto, se desconocen sus características sociodemográficas, qué hacen en su tiempo de ocio estival, cuál es su nivel de satisfacción o su grado de fidelidad con el destino. Esta debilidad en el conocimiento científico a nivel local dificulta la elaboración de estrategias turísticas municipales.

\section{DIFICULTADES METODOLÓGICAS Y TÉCNICAS A LA LUZ DE UN ESTU- DIO DE CASO}

La investigación que nos sirve de caso-ejemplo sobre el que mostrar las soluciones adoptadas para evitar las citadas dificultades es el proyecto Turismo residencial: análisis de la demanda y propuestas de reestructuración para destinos consolidados ${ }^{4}$ (en adelante TURVERCON).

Los objetivos de este proyecto eran dos. El primero de ellos se orientaba al análisis y diagnóstico del modelo turístico-residencial de la Costa Blanca. Este objetivo se cubrió mediante la construcción de una serie de indicadores a partir de datos secundarios, entrevistas semiestructuradas a informadores claves y dinámicas de grupo.

El segundo de los objetivos se centraba en el estudio del perfil socio-turístico del veraneante residencial en la Costa Blanca (en adelante VRCB) mediante la aplicación de una encuesta a veraneantes españoles en cinco municipios de la Costa Blanca: Benidorm, Altea, Denia, Santa Pola y Torrevieja. Su elección se rigió por motivos de su especialización en el turismo de veraneo de sol y playa (García-Andreu y Rodes, 2004; Mazón, 2006), el elevado número de plazas extrahoteleras que ofrecen a la demanda turística (EXCELTUR, 2005) y la pretensión de abarcar geográficamente la Costa Blanca de Norte a Sur. La toma de datos se realizó durante las dos últimas semanas del mes de julio de 2008 y de manera simultánea en las poblaciones seleccionadas. A nivel operativo, el estudio definió a los turistas residenciales veraneantes como aquellos que se desplazan por periodos de tiempo de entre una semana y tres meses durante el periodo estival, y que utilizan como alojamiento turístico su segunda residencia, la de amigos o familiares, viviendas alquiladas o en régimen de tiempo compartido.

Si bien este segundo objetivo habría de cubrirse con una encuesta a los turistas residenciales veraneantes, esta técnica ofrecía dificultades importantes desde su inicio. Se desconocían los datos básicos para la realización de un muestreo «clásico»: el tamaño de la población objeto de estudio a nivel municipal, así como sus características sociodemográficas, su distribución temporal (fechas de permanencia en el destino) y su distribución espacial, dada la morfología extensa de ocupación del espacio.

\subsection{Dificultades en la estimación del tamaño de la población}

En primer lugar, el equipo de investigación de TURVERCOM se enfrentó a la falta de datos a escala local sobre el número de veraneantes españoles que escogían el alojamiento

4 Referencia SEJ2005-04305 «Turismo residencial: análisis de la demanda y propuestas de reestructuración para los destinos consolidados». Ministerio de Educación (Gobierno de España) en su convocatoria de Investigación Fundamental No Orientada 2005-2008. 
residencial para su veraneo en la Costa Blanca. La solución adoptada en TURVERCOM se apoyó en cuatro referencias trianguladas: a) número de viviendas de potencial uso turístico; b) cálculos sobre la producción de residuos sólidos urbanos; c) entrevistas en profundidad a informantes clave; y d) estimaciones estadísticas sobre encuestas turísticas (datos oficiales) en origen.

a) Número de viviendas de potencial uso turístico. Diversos intentos de cuantificar a los turistas residenciales veraneantes han empleado el concepto de Viviendas de Potencial Uso Turístico (VPUT) (Chasco, 1996; Cortina, Martínez y Varela, 2002; EXCELTUR, 2005). Las VPUT se definen como aquellas que en el Censo de Población y Viviendas (Instituto Nacional de Estadística, 2004) se contabilizan como secundarias o vacías en municipios turísticos. A partir de estos datos, investigaciones previas calcularon las siguientes plazas extra-hoteleras para los cinco municipios investigados: Altea 22.287, Benidorm 111.517; Denia 78.299, Santa Pola 90.335 y Torrevieja 274.686. (García-Andreu, 2008).

No obstante la consideración de las VPUT, como base para calcular cuántos turistas residenciales hay en un destino turístico, acarrea fuertes imprecisiones y problemas.

Primero, solo se dispone del dato de VPUT cada 10 años, pues es la periodicidad con que se realiza el Censo Nacional de Población y Viviendas -el último disponible a fecha de realización de la investigación era el del año 2001. Desde esa fecha, el número de segundas residencias ha aumentado considerablemente en la Costa Blanca (Greenpeace, 2010; Naredo y Montiel, 2011) lo que resta fiabilidad a los datos censales. Segundo, para la aproximación a dicho cálculo se aplica a las VPUT un coeficiente de ocupación que no está basado en datos reales: se desconoce la composición (cuantía, características sociodemográficas, vinculación de los miembros...) del grupo que viaja y se aloja en esas viviendas en verano. Tercero, tampoco se conoce durante cuánto tiempo son usadas, ni en qué período exacto. Por último, también se ignora el país de procedencia de los ocupantes. FRONTUR (2010: 44) señala que el 55\% de las pernoctaciones de turistas extranjeros son realizadas en la oferta extra-hotelera, pero a escala local no existe información que permita conocer su nacionalidad. Estos problemas hacen que el dato de VPUT no pueda considerarse por sí solo un estimador adecuado de la población turístico-residencial española. Por todo ello, para la estimación del universo poblacional se buscaron vías alternativas que ofreciesen la posibilidad de triangular los datos de base.

b) Cálculos sobre la producción de residuos sólidos urbanos. A este dato se sumaron las estimaciones de visitantes a través de los datos de residuos sólidos urbanos (RSU) producidos por habitante. En un proyecto anterior, este equipo había calculado el número de visitantes -sin realizarse una diferenciación según nacionalidad del visitante- en estos cinco destinos mediante los datos semanales proporcionados por las empresas de recogidas de basuras orgánicas domésticas. El cálculo consideraba el peso de los RSU en el municipio, sus fechas de producción y un coeficiente de producción de RSU por habitante (Aledo y García-Andreu, 2007). Según los cálculos obtenidos, para la segunda semana del mes de julio de 2005 en Torrevieja 
se contabilizaban 465.672 personas, mientras que el INE daba 84.348 censadas en ese año; 171.053 en Denia frente a las 40.601 censadas; en Santa Pola 257.238 según los cálculos del proyecto frente a las 25.494 que aparecen en el Censo; y en Altea 73.157 frente a los 21.154 residentes censados. Los datos referidos al municipio de Benidorm -que cuenta con una planta hotelera significativamente mayor que la del resto de municipios- tuvieron que ser desechados debido a la gran cantidad de basuras procedente de los restos de las comidas de los bufés de los hoteles que distorsionaban la ratio de $\mathrm{Kg} \mathrm{RSU/persona.}$

c) Entrevistas en profundidad a informantes clave. Seguidamente se llevaron a cabo una serie entrevistas semi-estructuradas a informantes siguiendo un muestreo intencional con el fin de recoger el conocimiento de expertos a fin de discernir el porcentaje de VRCB en cada uno de los destinos seleccionados. Se realizaron un total de 15 entrevistas en profundidad con el objeto de afinar los datos secundarios de RSU. Estos informantes fueron considerados estratégicos por su acceso a información relevante para estimar la población veraneante en el destino. En concreto, se entrevistaron técnicos de Ayuntamientos, cargos públicos con responsabilidad en turismo y urbanismo (Ayuntamientos y Diputación Provincial), responsables de asociaciones de empresarios turísticos y representantes de asociaciones de vecinos. Las conclusiones de las entrevistas realizadas apuntaban a un universo estimado superior a los 50.000 VRCB en los destinos escogidos -excepto para el municipio de Altea, que se le calculaba en torno a 25.000 veraneantes.

d) Estimaciones estadísticas sobre encuestas turísticas (datos oficiales) en origen. La aproximación al tamaño poblacional de referencia se completó con cálculos realizados sobre los microdatos del Instituto de Estudios Turísticos, obtenidos en la encuesta en origen FAMILITUR 2008 para el destino Costa Blanca. La inferencia realizada a partir de los datos aportados por esta encuesta mostraba una estimación de 774.000 personas residentes en España que habían realizado al menos un viaje de vacaciones de verano en alojamiento residencial a la Costa Blanca en el año 2008. Estas estimaciones proceden de la muestra de FAMILITUR 2008, formada por 14.274 hogares con 38.584 individuos. En FAMILITUR 2008, 613 individuos, procedentes de 268 hogares, habían realizado un viaje de vacaciones de verano residenciales a la Costa Blanca. Al tratarse de una muestra compleja, el error de muestreo para el número de turistas residenciales españoles en la Costa Blanca, es difícil de calcular, pero se puede estimar, de forma aproximada, a través de la fórmula del muestreo aleatorio simple (Abascal y Grande, 2005: 75:78:89), que lo sitúa en el 0,21\% de la población residente en España $(0,21 \times 44.764 .076= \pm$ 94.005 personas). Por tanto, a partir de FAMILITUR se infieren unas $774.000 \pm$ 94.000 personas, residentes en España, que han realizado vacaciones de verano en la Costa Blanca en alojamiento residencial.

Según FAMILITUR 2008, los 5 municipios tienen un volumen estimado del $50 \%$ del total de VRCB. A pesar del limitado número de casos sobre los que inferir, los cálculos arrojan a nivel municipal 138.100 veraneantes residenciales en Benidorm, en Denia 45.430, en Santa Pola 60.577, en Torrevieja 116.655 y en Altea 10.506. Si bien ha de 
reconocerse la debilidad técnica de esta distribución municipal basada en la encuesta oficial de referencia en origen, la triangulación realizada (VPUT, cálculos sobre los RSU y entrevistas a informantes clave) aportaba un extra de fiabilidad que permitía avanzar en el conocimiento del universo con unas garantías mínimas para realizar la encuesta y, así, cubrir nuestro segundo objetivo en TURVERCON. Considerando la información de las cuatro fuentes de triangulación citadas, pudimos establecer con seguridad razonable que el conjunto de los cinco municipio elegidos para definir el destino turístico a analizar superaba con creces el mínimo de 100.000 personas, límite inferior del universo infinito en los estudios estadísticos.

Para reducir al máximo posible el error de muestreo en cada localidad elegida, y a la vista de la disponibilidad de recursos del proyecto, se decidió utilizar un tamaño de muestra de 400 individuos en las localidades de Denia, Benidorm, Santa Pola y Torrevieja. Este tamaño de muestra, calculado bajo el supuesto de muestreo aleatorio simple, implicaba un error máximo de muestreo de un 5\%, para un nivel de confianza del 95,5\%. Para Altea se estableció un tamaño muestral de 200 unidades, debido principalmente a un universo más pequeño (se estimaron 10.000 individuos y se aceptó un error máximo del 7\%), que se puso de manifiesto en las dificultades encontradas por los entrevistadores para completar el número total de entrevistas asignadas (Abascal y Grande, 2005; Cea d'Ancona, 1996; Henry, 1990).

\subsection{Dificultades en la selección de la muestra}

La segunda dificultad a subsanar fue el formato de distribución muestral. El desconocimiento de las características cualitativas y cuantitativas de la población imposibilita el establecimiento de cuotas, estratos u otros criterios de selección muestral. La dispersión de las viviendas secundarias por un amplio territorio dificulta la utilización de rutas aleatorias como método de muestreo. Además, estas residencias no se usan de forma permanente ni regular en el tiempo, lo que impide la correcta elección de la vivienda. Asimismo, los patrones de movilidad y ocio de los veraneantes dificultan la realización de las entrevistas en los domicilios, ya que durante las vacaciones la permanencia en las viviendas se reduce ante las opciones de ir a la playa, pasear o visitar otros destinos.

La solución adoptada para este problema consistió en recabar información sobre las zonas urbanas de mayor afluencia de veraneantes. Para ello se realizaron cinco entrevistas por municipio a informantes clave empleando los planos municipales para que los entrevistados pudieran localizar las áreas de mayor afluencia turístico-peatonal, así como los horarios de más elevada frecuencia de paso. La información obtenida en los mapas se contrastó con observación sobre el terreno. Los responsables del trabajo de campo se desplazaron a los municipios para comprobar que los mapas aportados por los informantes clave respondían a la realidad. Estos viajes al campo se aprovecharon para realizar un pre-test del cuestionario, que sirvió para ajustar su redacción y su tiempo de realización.

Con esta información se establecieron puntos y rutas de muestreo en cada localidad en los que existía una alta presencia o trasiego de personas durante la época estival. Considerando que los turistas residenciales que veranean en estos núcleos tienen como motivo de referencia el «sol y playa», se diseñó el trabajo de campo de la siguiente forma. Las rutas 
se establecieron en las playas, donde se aplicó el 70\% de los cuestionarios. El 30\% restante se recogió en puntos de muestreo definidos como lugares de elevado trasiego humano en cada localidad: determinadas esquinas en calles comerciales de zonas céntricas, plazas de áreas comerciales y/o de establecimientos hosteleros, puntos determinados en paseos marítimos o zonas peatonales y accesos a centros comerciales.

Además, se fijaron unos intervalos horarios en cada uno de dichos puntos y rutas para respetar, en lo posible, el requerimiento probabilístico de que a mayor presencia o trasiego, mayor fuese el número de encuestados. Por tanto, las franjas horarias se definieron en función de la densidad humana tanto en rutas como en puntos de muestreo. Así, de 10:00 a 14:30 y de 17:00 a 20:00 horas se realizaron los cuestionarios en las playas, y a partir de las 20:00 horas en los puntos de muestreo. Para la selección de la unidad muestral se estableció el criterio sistemático de entrevistar al octavo individuo en ruta o punto de muestreo, después de finalizar una entrevista.

\subsection{Dificultades relacionadas con sesgos conocidos}

Los sesgos que aparecen al seleccionar individuos en destino, como en el caso de TURVERCON, supusieron una tercera dificultad. En concreto, se detectaron dos sesgos que podrían ser influyentes en la fiabilidad de la información recogida para análisis: el sesgo por duración de la estancia y el sesgo por edad del encuestado. Estos sesgos se pueden corregir de diferentes formas, bien modificando el método de selección o corrigiendo el peso de los individuos en la muestra durante el análisis posterior.

Respecto al sesgo por duración de la estancia, la bibliografía especializada señala que en las encuestas en destino los turistas que permanecen más tiempo en el lugar tienen más posibilidades de ser entrevistados (lenght-of-staybias) (Loomis, 2007; Lucas, 1963; Nowell, Evans y McDonald, 1988; Ojeda, Cristóbal y Alcalá, 2011). Este sesgo es relevante en esta investigación, pues se estima que casi la mitad de los viajes de veraneo residencial a la Costa Blanca duran entre una y dos semanas, y la otra mitad entre dos semanas y tres meses ${ }^{5}$. Este sesgo puede ser corregido durante el análisis de los datos, ponderando los registros de cada individuo con el valor inverso de la duración de su estancia (Loomis, 2007:46, Lucas, 1963; Nowell, Evans y McDonald, 1988: 368).

Cuando la distribución de los visitantes de un destino a lo largo del periodo de tiempo estudiado es muy variable, un plan de muestreo estable tendrá un sesgo hacia los visitantes de los periodos con menor afluencia. El sesgo temporal, en el caso que nos ocupa, se puede corregir utilizando la distribución temporal mensual de los viajes de veraneo residencial de los españoles a la Costa Blanca (agregación de todos los municipios del destino). Esta distribución temporal se obtuvo a partir de las estadísticas oficiales en origen (FAMILITUR, 2008). Esta corrección del sesgo temporal requiere que, en las entrevistas en destino, se pregunte al entrevistado por la fecha de inicio y final de su viaje para poder llevar a cabo una correcta triangulación con los datos de FAMILITUR (encuesta/panel en origen) y adjudicar cada viaje al mes correspondiente según la fecha final del viaje. Se trata, por tanto, de llevar a cabo un ejercicio de triangulación entre

\footnotetext{
5 Elaboración propia a partir de microdatos de Familitur 2008.
} 
encuestas en origen y en destino, a nivel agregado, para calcular los pesos que ajusten la distribución temporal de la muestra en destino a la distribución temporal (por meses) del número de turistas residenciales españoles en la Costa Blanca, según las encuestas oficiales en origen.

Respecto al sesgo por edad del entrevistado, Ortega (1990: 361) advierte que en determinadas muestras seleccionadas por un procedimiento aleatorio simple, no es inusual que los jóvenes estén menos representados que el resto de la población. Por tanto, hay ciertas edades y colectivos que son más fácilmente entrevistables. TURVERCON controló este sesgo mediante dos procedimientos. Por una parte, se instruyó a los entrevistadores para elegir aleatoriamente al entrevistado cuando abordaran a un grupo de turistas. Por otra parte, en el cuestionario de TURVERCON se incluyeron preguntas sobre la edad y sexo de los miembros del grupo de viaje que acompañaba a cada entrevistado. De esta forma, se podría comparar luego la distribución de la edad y sexo de todos los miembros de los grupos de viaje con la distribución de la edad y sexo de los entrevistados.

En la muestra de TURVERCON se detectó que, tal como indicaba Ortega (1990), los menores de 30 años aparecían infrarrepresentados, mientras que las personas de entre 36 y 65 años estaban ligeramente sobre-representadas. Por tanto, a partir de la información sobre el grupo de viaje, para el análisis se ponderó el número de entrevistados en función de su edad, realizando los cálculos para corregir este sesgo (Domínguez, Aledo y Roig, 2011).

En este punto hay que recomendar, para estudios de similares características, la inclusión de algunas preguntas en los cuestionarios a realizar en destino que estén formuladas también, y de la misma forma, en la encuesta de referencia en origen (FAMILITUR para este caso). Edad, sexo, región/provincia de procedencia, duración de la estancia (para minimizar el sesgo por duración de la estancia) y tipo de alojamiento utilizado podrían ser algunas de ellas; así es posible ponderar y comparar resultados entre ambas encuestas.

Esta corrección de sesgos no es frecuente en las encuestas a visitantes en destino llevados a cabo en España, en las cuales los datos son analizados directamente de la muestra (Sanz, 2008; Roca, Villares y Ortego, 2009). En cambio, en las estadísticas oficiales (INE; IET), en origen y en frontera sí que se ponderan los individuos para que la muestra sea representativa de la población, y se aplican coeficientes correctores para corregir éstos y otros sesgos como, por ejemplo, el efecto olvido cuando se le pregunta a un entrevistado por viajes realizados hace 1, 2 ó 3 meses (FAMILITUR).

\subsection{Dificultades relacionadas con la recogida de información}

Por último, la aplicación de una entrevista durante el periodo estival, en momentos y lugares de ocio y esparcimiento, supuso también un reto para la calidad del dato recogido. Previo a la toma de datos, como es normativo en encuestas que se ejecutan por primera vez, la herramienta se puso a prueba realizando un pre-test de 20 casos en cada municipio; 100 en total. A la vista de los resultados de este pre-test, se modificó la redacción de algunas preguntas para ajustar el tiempo de realización. Así, cada cuestionario se realizaría en un máximo de 20 minutos para adaptarse a las condiciones de la entrevista al realizarse en la playa o en zonas de paseo. 
Los clásicos problemas relacionados con la obtención del dato (Alvira, 2004; Díaz de Rada, 2001; Núñez, 2005) fueron especialmente estudiados en el caso de TURVERCON, a sabiendas de sus particularidades de diseño y de las dificultades que entrañaba el trabajo de campo en destinos turísticos. Así, se cuidó la selección de los encuestadores, los posibles déficits de calidad devenidos de las características personales de informante y encuestador y sobre el registro y grabación de los datos. De esta manera, se prestó especial interés en la consecución del dato intentando una máxima fiabilidad del mismo para compensar, en la medida de lo posible, otras dificultades a las que se enfrentaba esta investigación.

Los encuestadores se seleccionaron entre estudiantes de último curso de la licenciatura de sociología de la Universidad de Alicante. Recibieron un curso de 30 horas en el que se les entrenaba en las especificidades de la entrevista estructurada y la comprensión del cuestionario. Los encuestadores se dividieron en 5 grupos de trabajo con su correspondiente supervisor; cada grupo realizaba diarias puestas en común sobre la casuística del trabajo de campo, y cada cinco días una reunión de todos los grupos con los directores de la encuesta para, según la información de campo, ajustar el trabajo a las particularidades de los puntos y rutas de muestreo.

Entrevistador y entrevistado fueron controlados por los medios clásicos. El primero, solicitando su número de teléfono en el cuestionario, con la finalidad de evaluar la calidad del trabajo realizado. Además, permutas de entrevistadores entre ubicaciones (puntos y rutas de muestreo) y municipios, pretendieron diluir posibles sesgos en la toma de datos, vinculados a las diferentes capacidades o actitudes personales en el trabajo realizado. Respecto al control del entrevistado, se incluyeron en el cuestionario preguntas para la verificación de la coherencia informativa. Además, durante su formación específica, los entrevistadores fueron entrenados para detectar, mediante estas preguntas y la comunicación no verbal, posibles olvidos del entrevistado y su sinceridad.

\section{CONTRASTACIÓN DE LAMETODOLOGÍA: RESULTADOS DE LAENCUESTA EN DESTINO FRENTE A LA ENCUESTA OFICIAL DE REFERENCIA}

Para cumplir con el objetivo de esta investigación, que se aproxima al estudio de una población elusiva con instrumentos metodológicos caracterizados por una alta flexibilidad y una constante triangulación de datos, se hace necesario contrastar la fiabilidad de toda esta estrategia. Para ello, se procede seguidamente a comparar el perfil del VRCB obtenido a partir de la encuesta en destino (TURVERCON) con el elaborado a partir de la encuesta en origen (FAMILITUR 2008). Con esta operación se pretende conocer en qué medida se pueden extrapolar los resultados de TURVERCON al conjunto del universo estudiado (VRCB en 2008).

En primer lugar, para la encuesta en destino se han ponderado los registros de cada municipio elegido en función del volumen de VRCB estimado para cada uno de ellos a partir de FAMILITUR 2008. Una vez preparadas las bases de datos, se procede a comprobar la representatividad de la muestra TURVERCON, comparando los resultados obtenidos a partir de esta encuesta con los de la submuestra de FAMILITUR VRCB. Para ello se han tabulado una serie de variables, seleccionadas de ambos cuestionarios por formularse exactamente del mismo modo. 
Las preguntas del cuestionario en destino que se pueden contrastar con las de FAMILITUR son de dos tipos: 1) preguntas relativas a todas las personas que viajan con el entrevistado (grupo de viaje): sexo y edad ${ }^{6} ; 2$ ) Preguntas relativas solo al entrevistado ${ }^{7}$ : estado civil, nivel de estudios, provincia de origen, duración del viaje y situación laboral.

1) En cuanto a las preguntas referidas a todo el grupo de viaje, la comparación de sexo y edad (Anexo, Tabla 1) de los componentes del grupo de viaje arroja diferencias mínimas entre TURVERCON y FAMILITUR, que no son estadísticamente significativas y caben dentro de los márgenes de error admitidos en cada una de las dos encuestas.

2) Tampoco se encuentran diferencias significativas en las preguntas relativas al entrevistado. Respecto al estado civil (Anexo, Tabla 2), las diferencias más importantes aparecen en el porcentaje de casados, aunque no exceden los márgenes de error admitidos. En cuanto al nivel de estudios (Anexo, Tabla 3) del entrevistado, las diferencias más elevadas se encuentran en la categoría que agrupa a los «estudios primarios», aunque también dentro de los márgenes de error admitido ${ }^{8}$. En la provincia de residencia del viajero (Anexo, Tabla 4), las diferencias encontradas están dentro del error asumible, concentrando entre Madrid y Alicante a la mitad de los viajeros. La distribución de los resultados por categorías en estas variables también es similar.

En el tiempo de estancia en el destino (Anexo, Tabla 5) se han agrupado en dos las diferentes categorías de respuesta, dada la fuerte predominancia de las estancias cortas (entre una semana y 15 días). La coincidencia con FAMILITUR es prácticamente total. Aunque, como se ha señalado con anterioridad, en TURVERCON podría esperarse un sesgo hacia las estancias con mayor duración, sin embargo, el hecho de haber realizado las entrevistas en el mes de julio, que muestra una duración de estancia menor que en agosto (FAMILITUR, 2008), ha provocado un sesgo hacia las estancias más cortas, que ha compensado el sesgo por duración de la estancia.

6 De estas preguntas se dispone de 6.567 registros en TURVERCON, procedentes de 1.801 entrevistas válidas, lo que supone un error de muestreo máximo aproximado de $2,4 \%$. Estos registros se pueden contrastar con la submuestra de 648 viajes VRCB6 de FAMILITUR 2008, ya que aquí cada viaje se contabiliza como una persona que se desplaza. Estos 648 registros de FAMILITUR proceden de 268 hogares diferentes, lo que implica un error máximo de muestreo aproximado 6,1\%, para un nivel de confianza del 95,5\% con un muestreo aleatorio simple.

7 De estas preguntas se dispone de 1.801 registros en destino, por lo que el error máximo aproximado en TURVERCOM sigue siendo de $\pm 2,4 \%$. Estas preguntas se pueden contrastar con la submuestra de FAMILITUR VRCB6 de mayores de 15 años, obtenida de 264 hogares, lo que supone un error aproximado de muestreo de, máximo, $\pm 6,1 \%$. La situación ideal para esta contrastación se daría con la elección de universos (destinos y tipos de viajes) cuyo tamaño muestral en FAMILITUR no sea menor de 400 hogares.

8 Aquí se aprecia un posible sesgo hacia un mayor nivel de estudios declarado en la muestra en destino, que estaría causado por el tipo de entrevistador utilizado en TURVERCON (estudiantes universitarios) y también por el entrevistado (tendencia a declarar un nivel de estudios superior al real, cuando unos estudiantes universitarios le entrevistan en la calle). Este sesgo no se produce en la encuesta en origen (FAMILITUR), ya que se trata de un panel de hogares que accede a colaborar de forma permanente y se realiza un mayor control y verificación de los datos reales sobre los entrevistados. 
Por último, en la situación laboral del encuestado (Anexo, Tabla 6), las categorías comparables muestran resultados muy parecidos, siempre dentro del margen de error asumido.

Se observa, por tanto, que los resultados de ambas encuestas, en términos generales, son muy similares. Las diferencias más importantes halladas en algunas categorías podrían encontrarse en su diferente formato (TURVERCON a pie de calle, FAMILITUR tipo panel) y en las circunstancias propias de la ejecución de cada formato (por ejemplo, el formato panel facilita la «inercia» en la respuesta, pues se pregunta a la misma muestra sobre las mismas circunstancias o asuntos, aunque en tiempos diferentes).

\section{CONCLUSIONES}

Desde el punto de vista epistemológico, huir de las dificultades de las poblaciones elusivas significa un freno para el conocimiento de objetos de estudio que, como en el caso del turismo residencial, pueden ser trascendentes para la sostenibilidad del territorio y de las comunidades locales. A lo largo del texto se han explicado las dificultades que se han ido encontrando a lo largo de la investigación y cómo se han subsanado. Así, en este trabajo los objetivos se alcanzan por medio de una estrategia flexible, con una solución a medida para cada uno de los problemas hallados. La última parte de este artículo demuestra que los resultados obtenidos mediante esta estrategia flexible y adaptativa de investigación ofrecen niveles de confiabilidad similares a los más ortodoxos.

Frente a aquella investigación con fuerte carácter deductivo, positivista y autorreferencial, se plantea aquí, de fondo, la necesidad de una apuesta por la investigación centrada en el interés y la necesidad de cubrir unos objetivos. Una investigación donde los problemas operativos que plantee el objeto no sean un freno apriorístico para el conocimiento funcional, que sirva para la solución de problemas sociales reales más que para multiplicar la cantidad de publicaciones científicas mediante la replicación de modelos que, a menudo, difícilmente responden a la realidad.

La motivación inicial del estudio-caso aquí presentado fue la aportación de conocimiento estratégico sobre unos procesos y poblaciones extremadamente importantes para amplias zonas litorales de España. La elusividad característica de la población turísticoresidencial impedía el diseño de un muestreo clásico.

La información necesaria para desarrollar el trabajo de encuesta se consiguió mediante una estrategia de triangulación informativa mixta, cualitativa y cuantitativa. Cada una de las cuatro fuentes o técnicas empleadas ofreció información que complementaba la de las demás, de forma que pudo realizarse el cálculo aproximado del universo poblacional. A continuación, la selección muestral se diseñó siguiendo la misma estrategia de triangulación, con entrevistas a informantes clave y observación de campo, al objeto de adaptar el trabajo a la realidad y los comportamientos de los encuestados. Estudiando los sesgos de las encuestas en destino, se controlaron en lo posible (según las limitaciones propias del proyecto) aquéllos que afectaban en mayor medida al estudio realizado. No obstante, a la luz de la experiencia recabada, también se realizan recomendaciones para la ejecución de estudios en circunstancias similares al expuesto.

Una investigación social no es un objeto cerrado, neutral y exclusivamente técnico. Una investigación es un proceso social y, como tal, influenciado por su entorno socio- 
político. En el caso que nos ocupa, la producción de conocimiento sobre el turismo residencial está condicionada por la particular gobernanza del urbanismo en los municipios españoles y por el marco de crisis económica en el que se encuentran (Naredo y Montiel, 2011).

Este marco requiere, por tanto, una reflexión por parte de la sociología del turismo acerca de la aplicación del instrumento de encuestación, de las teorías que permiten elaborar hipótesis y su contrastación para la formulación del cuestionario, del trabajo de campo y recogida de datos, del proceso de análisis estadístico y del proceso inductivo de formulación de inferencias. En esta línea reflexiva cabe decir que, mientras los manuales sobre metodologías cualitativas se han preocupado por mostrar el proceso social de investigación, los proyectos cuantitativos generalmente han reducido la descripción de la investigación a la «ficha de la encuesta» y dicen poco de la «trastienda de la encuesta» (Sautu, 2009). Se pone en este caso de manifiesto lo que podríamos denominar la arrogancia del neopositivismo y esa creencia mítica de que el dato está ahí fuera, que no se construye socialmente y que con un buen cuestionario y un potente aparato matemático-analítico la verdad aflora de forma directa y objetiva (Wainerman, 2009). Pero cualquier investigador que sea honesto y que tenga un mínimo del requisito inexcusable de reflexividad sabe que una investigación no es un objeto cerrado, neutral y técnico, sino un proceso social y, como tal, sujeto a innumerables vicisitudes que sitúan al ejercicio de la investigación en el mundo posmoderno de la incertidumbre.

En esa construcción, en el proceso social de la investigación y de producción de sus resultados, influyen toda una serie de imponderables. Hay pocos manuales que aborden estos imponderables (Alvira, 2004) pero una parte importante de la tarea científica y del esfuerzo del investigador se dedica a superarlos (Camacho, Romero de Loera, Valero y Camacho, 2000). Y esa superación pasa por un ejercicio reflexivo, por entender la investigación como un proceso, por el empleo de diseños abiertos y emergentes y por una flexibilidad metodológica que si bien no está exenta de riesgos es un requisito de cientificidad en un mundo dominado por el cambio.

\section{REFERENCIAS BIBLIOGRÁFICAS}

ABASCAL, E. y GRANDE, I. (2005): Análisis de encuestas. Madrid, ESIC Editorial. ALEDO, A. y GARCÍA-ANDREU, H. (2007): Métodos de estimación y proyección de la población en municipios turísticos residenciales: La demanda del agua y sus previsiones. Generalitat Valenciana.

ALEDO, A. (2008): «De la tierra al suelo. La transformación del paisaje y el nuevo turismo residencial» Arbor, vol. 184, no 729, pp. 99-113.

ALMENAR, R., BONO, E. y GARCÍA, E. (2000): La sostenibilidad del desarrollo: el caso valenciano. Valencia, Universidad de Valencia.

ALVIRA, F. (2004): La encuesta: una perspectiva general metodológica. Madrid, Centro de Investigaciones Sociológicas.

BEN-HAIM, Y., ZACKSENHOUS, M., KEREN, C. y DACSO, C. (2009): «Do we know how to set decision thresholds for diabetes?», Medical Hypotheses, vol. 73, $\mathrm{n}^{\circ} 2$, pp. 189-193. 
CAMACHO, C., ROMERO DE LOERA, B., VALERO, S. y CAMACHO, C. (2000): «¿Cómo hacen encuestas los encuestadores?», Metodología de Encuestas, vol. 2, no 1, pp. 117-133.

CASADO-DIAZ, M.A. (1999): «Socio-demographic impacts of residential tourism: A case study of Torrevieja, Spain», The International Journal of Tourism Research, vol. $1, n^{\circ} 4$, pp. 223-237.

CEA D’ANCONA, M.A. (1996): Metodología cuantitativa: Estrategias y técnicas de investigación social. Madrid, Síntesis.

CHASCO, C. (1996): "Analysis of the important gap existent between the official tourism statistical and the real ones», 20th Conference on Regional and Urban Statistics, SCORUS. Madrid.

COOPER, G., KING, A. y RETTIE, R. (2009): Sociological objects: Reconfiguration of social theory. Abingdon Oxon, Ashgate Publishing Group.

CORTINA, F., MARTÍNEZ, A. y VARELA, B. (2002): "Aproximación a la investigación del alojamiento privado de uso turístico», Estudios Turísticos, n ${ }^{\circ} 151, \mathrm{pp}$. 87-98.

CRESWELL, J.W. y PLANO, V.L. (2011): Designing and Conducting Mixed Methods Research .SAGE Publications.

DAVID, B. y SNIJDERS, T.A.B. (2002): «Estimating the size of the homeless population in Budapest, Hungary», Quality \& Quantity, vol. 36, n 3, pp. 291-303.

DÍAZ DE RADA, V. (2001): Diseño y elaboración de cuestionarios para la investigación comercial. Pozuelo de Alarcón, ESIC.

DÍAZ DE RADA, V. (2006): «Las incidencias en la investigación con encuestas», Metodología de Encuestas, vol. 8, nº 1, pp. 3-10.

DOMÍNGUEZ, J.A., ALEDO, A. y ROIG, B. (2011): «The identity of sociology or what to do when the universe is unknown: qualitative solutions against the quantitative obsession», Spatial and Organizational Dynamics Discussion, papers $\mathrm{n}^{\circ}$ 5, pp. 7-23.

DURKHEIM, E. (1973): Les règles de la méthode sociologique (18 ed.). Paris, Presses Universitaires de France.

EXCELTUR. (2005): Impacto de los modelos de desarrollo turístico en el litoral mediterráneo y las islas.

FERNÁNDEZ, M. (2003): «Criterios de calidad en la investigación social: la producción de datos sociales», Empiria: Revista de metodología de ciencias sociales, $\mathrm{n}^{\circ}$ 6, pp. 47-78.

FERNÁNDEZ, R. (2006): «El Tsunami Urbanizador Español y Mundial», Revista Habitat. Ciudades para un futuro más sostenible, vol. 38-39. Disponible en http://habitat. aq.upm.es/boletin/n38/arfer.html.

GARCÍA-ANDREU, H. y RODES, J. (2004): «Análisis y comparativa de cinco municipios turísticos de la Costa Blanca», en Turismo, ocio y deporte. A Coruña, Universidad de La Coruña, pp. 377-392.

GARCÍA-ANDREU, H. (2005): «Un acercamiento al concepto del turismo residencial», en Turismo residencial y cambio social: nuevas perspectivas teóricas y empíricas. Alicante, CAM - Universidad de Alicante, pp. 55-70. 
GARCÍA-ANDREU, H. (2008): Sociedad, turismo y medio ambiente: una propuesta desde la sociología para el diagnóstico y reorientación de los municipios turísticoresidenciales del litoral español, Tesis doctoral, Universidad de Alicante.

GREENPEACE. (2010): Destrucción a toda costa 2010. Disponible en http://www.greenpeace.org/espana/Global/espana/report/other/100709-04.pdf

HAMEL, J. (1992): «On the status of singularity in sociology», Current Sociology / La Sociologie Contemporaine, vol. 40, $\mathrm{n}^{\circ}$ 1, pp. 99-119.

HARRISON, R.L. y REILLY, T.M. (2011): «Mixed methods designs in marketing research», Qualitative Market Research: An International Journal, vol. 14, $\mathrm{n}^{\circ} 1$, pp. 7-26.

HECKMANN, F. (2004): «Illegal migration: What can we know and what can we explain? The case of Germany», International Migration Review, vol. 38, $\mathrm{n}^{\circ} 3, \mathrm{pp}$. 1103-1125.

HENDERSON, T.L., SIROIS, M., CHIA-CHEN, A., AIRRIESS, C., SWANSON, D.A. y BANKS, D. (2009): «After a disaster: Lessons in survey methodology from hurricane Katrina», Population Research and Policy Review, vol. 28, n 1, pp. 67-92.

HENRY, G.T. (1990): Practical sampling. London, Sage.

HO, Wing-Chung. (2008): «Understanding the subjective point of view: Methodological implications of the Schutz-Parsons debate». Human Studies, vol. 31, nº 4, pp. 383-397.

HUETE, R. y MANTECÓN, A. (2011): «Más allá del turismo: movilidad residencial europea y nuevos núcleos urbanos», Boletín de la Asociación de Geógrafos Españoles, $\mathrm{n}^{\circ} 56, \mathrm{pp} .111-128$.

INSTITUTO NACIONAL DE ESTADÍSTICA (INE). (2004): Censo de Población y viviendas 2001.

INSTITUTO DE ESTUDIOS TURÍSTICOS. (2009): FAMILITUR 2008: Publicaciones anuales elaboradas por el instituto de estudios Turísticos sobre turismo nacional, Ministerio de Industria, Turismo y Comercio.

INSTITUTO DE ESTUDIOS TURÍSTICOS. (2010): FRONTUR: Movimientos turísticos en fronteras, Ministerio de Industria, Turismo y Comercio.

KARLSEN, J.E., OVERLAND, E.F. y KARLSEN, H. (2010): «Sociological contributions to future's theory building», Foresight, vol. 12, no 3 , pp. 59-72.

KUKLA, A. (2000): Social constructivism and the philosophy of science. London, Routledge.

LOOMIS, J (2007): «Correcting for on-site visitor sampling bias when estimating the regional economic effects of tourism», Tourism Economics, vol. 13, n 1, pp. 41-47.

LUCAS, R. C. (1963): «Bias in estimating recreationists' length of stay from sample interviews», Journal of Forestry, vol. 61, n 12, pp. 912-914.

MAINAR, A.J. y GRILLÓ, A.J. (2006): «Problemática en el desarrollo del trabajo de campo en investigación de mercado: Orígenes y soluciones», Metodología de Encuestas, vol. $8, \mathrm{n}^{\circ} 1$, pp. 17-21.

MAYO, R. y VELASCO, M.C. (2007): «Alojamiento privado de uso turístico desde el lado de la oferta», Índice - Revista de Estadística y Sociedad, n ${ }^{\circ} 24$, pp. 24-26.

MAZÓN, T. (2006): «Inquiring into residential tourism: The Costa Blanca case», Tourism and Hospitality Planning \& Development, vol. 3, n 2, pp. 89-97. 
NAREDO, J. M. y MONTIEL, A. (2011): El modelo inmobiliario español. Madrid, Editorial Icaria.

NOWELL, C., EVANS, M.A. y McDONALD, L. (1988): «Length of stay sampling in contingent valuation Studies», Land Economics, vol. 64, n 4, pp. 367-371.

NÚÑ̃Z, A. (2005): «Incidencias de la entrevista personal en la investigación mediante encuesta», Revista Española de Investigaciones Sociológicas, nº 109, pp. 219-236.

OBRADOR, P., CRANG, M. y TRAVLOU, P. (2009): Cultures of Mass tourism: doing Mediterranean in the Age of Banal Mobilities. Farnham, Ashgate.

OBSERVATORIO DE LA SOSTENIBILIDAD EN ESPAÑA (OSE). (2006): Cambios de ocupación del suelo de España. Madrid, Mundi-Prensa.

OJEDA, J.L., CRISTÓBAL, J.A. y ALCALÁ, J.T. (2011): «Biased data and its analysis», Boletín de Estadística e Investigación Operativa, vol. 27, n 2, pp. 91-101.

ORTEGA, E. (1990): Manual de investigación comercial. Madrid, Pirámide.

ORTIZ, G. (2009): Participación ciudadana, investigación social y desarrollo local: una propuesta metodológica aplicada a un municipio turístico-residencial, Tesis doctoral, Universidad de Alicante.

ROCA, E., VILLARES, M. y ORTEGO, M.I. (2009): «Assessing public perceptions on beach quality according to beach users' profile: A case study in the Costa Brava (Spain)», Tourism Management, vol. 30, n 4, pp. 598-607.

RODRÍGUEZ, V. y WARNES, T. (2002): «Los residentes europeos mayores en España: repercusiones socioeconómicas y territoriales», El campo de las ciencias y las artes, $\mathrm{n}^{\mathrm{o}} 139, \mathrm{pp} .123-148$.

ROGER, G. (2007): «Las Peculiaridades del Modelo Urbanístico Español: La Especulación Legalizada del Suelo», Algunas Claves de la Corrupción Urbanística en España. Fundación César Manrique.

SANZ, S. (2008): «Imagen global e intenciones futuras de comportamiento del turista de segunda residencia», Revista europea de dirección y economía de la empresa, vol. 17, $\mathrm{n}^{\circ} 4$, pp. 95-114.

SAUTU, R. (2009): «La trastienda de la investigación: el estudio de prácticas corruptas», Política y Sociedad, vol. 46, n 3, pp. 117-133.

SEIYAMA, K. (2006): «From empiricism to normative science», Sociological Theory and Methods, vol. 21, $\mathrm{n}^{\circ}$ 2, pp. 199-214.

SHARMA, B., DYER, P., CARTER, J. y GURSOY, D. (2008): «Exploring residents' perceptions of the social impacts of tourism on the sunshine coast, Australia», International Journal of Hospitality \& Tourism Administration, vol. 9, n 3, pp. 288-311.

SUDMAN, S., SIRKEN, M.G. y COWAN, C. D. (1988): «Sampling rare and elusive populations», Science, vol. 240, nº 4855, pp. 991-996.

TORRES, E. (2003): «El turismo residenciado y sus efectos en los destinos turísticos», Estudios Turísticos, $\mathrm{n}^{\circ}$ 155-156, pp. 45-70.

VERA, F. (1997): «la dimensión territorial y ambiental del turismo», en Análisis territorial del turismo: una nueva geografía del turismo. Barcelona, Editorial Ariel.

WAINERMAN, C. (2009): «De hachas vs bisturíes en la investigación social Cómo se hizo La vida cotidiana en las nuevas familias», Política y Sociedad, vol. 46, n 3, pp. $57-75$. 


\section{ANEXO}

Notas previas:

1. Todos los resultados mostrados se refieren a un supuesto de muestreo aleatorio simple, nivel de confianza $95 \%, \mathrm{~K}=1,96$.

2. El tamaño muestral en TURVERCOM es de 1.801 individuos. En FAMILITUR es de 268 individuos.

3. «Viajes» siempre se refiere al total de viajes de veraneantes residenciales a la Costa Blanca ponderado conforme a lo descrito en el cuerpo del artículo.

4. «Error» siempre se refiere al error de muestreo aproximado.

Tabla 1

SEXO Y EDAD DE LOS COMPONENTES DEL GRUPO DE VIAJE

\begin{tabular}{|c|c|c|c|c|c|}
\hline & & \multicolumn{2}{|c|}{ TURVERCON } & \multicolumn{2}{|c|}{ FAMILITUR } \\
\hline & & Viajes & Error & Viajes & Error \\
\hline \multirow{2}{*}{ Sexo } & Varón & $46,60 \%$ & $2,40 \%$ & $44,90 \%$ & $6,00 \%$ \\
\hline & Mujer & $53,40 \%$ & $2,40 \%$ & $55,10 \%$ & $6,00 \%$ \\
\hline \multirow{7}{*}{ Edad } & $0-10$ años & $11,90 \%$ & $1,50 \%$ & $15,50 \%$ & $4,30 \%$ \\
\hline & 11-20 años & $9,20 \%$ & $1,40 \%$ & $10,10 \%$ & $3,60 \%$ \\
\hline & 21-30 años & $11,90 \%$ & $1,50 \%$ & $11,60 \%$ & $3,80 \%$ \\
\hline & 31-40 años & $15,30 \%$ & $1,70 \%$ & $12,00 \%$ & $3,90 \%$ \\
\hline & 41-50 años & $15,00 \%$ & $1,70 \%$ & $15,30 \%$ & $4,30 \%$ \\
\hline & 51-60 años & $15,50 \%$ & $1,70 \%$ & $16,40 \%$ & $4,40 \%$ \\
\hline & $>60$ años & $21,00 \%$ & $1,90 \%$ & $19,10 \%$ & $4,70 \%$ \\
\hline Total & & $100 \%$ & & $100 \%$ & \\
\hline
\end{tabular}

Fuente: elaboración propia.

Tabla 2

ESTADO CIVIL DEL ENTREVISTADO (PORCENTAJES SOBRE VIAJES DE MAYORES DE 15 AÑOS)

\begin{tabular}{lcccc} 
& \multicolumn{2}{c}{ TURVERCON } & \multicolumn{2}{c}{ FAMILITUR } \\
\cline { 2 - 5 } & Viajes & Error & Viajes & Error \\
\hline Soltero/a & 24,5 & 2 & 28,1 & 5,4 \\
\hline Casado/a & 66,1 & 2,2 & 58,3 & 5,9 \\
\hline Viudo/a & 3,9 & 0,9 & 7,2 & 3,1 \\
\hline Divorciado/a & 1,8 & 0,6 & 2 & 1,7 \\
\hline Separado/a & 1,6 & 0,6 & 3,1 & 2,1 \\
\hline Pareja de hecho & 2,1 & 0,7 & 1,2 & 1,3 \\
\hline Total & $100 \%$ & & $100 \%$ & \\
\hline
\end{tabular}

Fuente: Elaboración propia. 
Tabla 3

NIVEL DE ESTUDIOS DEL ENTREVISTADO (PORCENTAJES SOBRE VIAJES DE MAYORES DE 15 AÑOS)

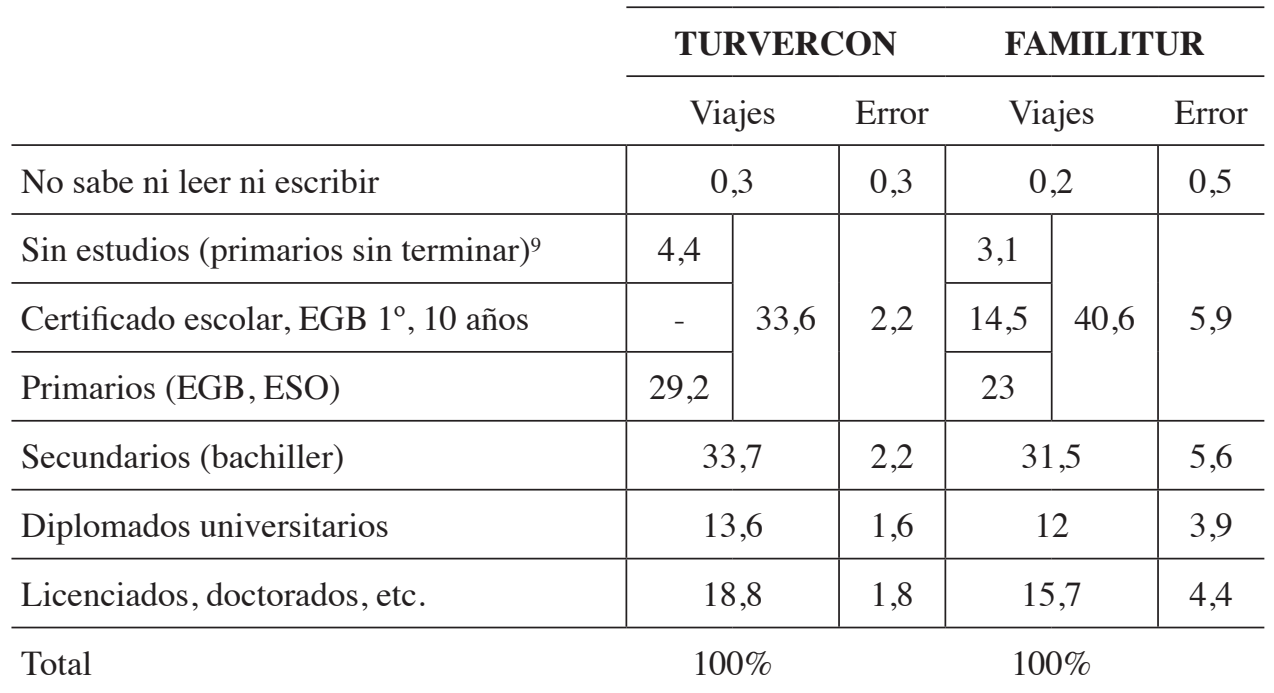

Fuente: elaboración propia.

Tabla 4

PROVINCIA DE PROCEDENCIA DEL ENTREVISTADO (PORCENTAJES SOBRE VIAJES DE MAYORES DE 15 AÑOS)

\begin{tabular}{lcccc} 
& \multicolumn{2}{c}{ TURVERCON } & \multicolumn{2}{c}{ FAMILITUR } \\
\cline { 2 - 5 } & Viajes & Error & Viajes & Error \\
\hline Alicante & 10,7 & 1,4 & 8,8 & 3,4 \\
\hline Madrid & 38,8 & 2,3 & 46,8 & 6 \\
\hline Valencia & 3,2 & 0,8 & 6,5 & 3 \\
\hline Resto prov. & 47,1 & 1,8 & 37,9 & 4,4 \\
\hline Total & $100 \%$ & & $100 \%$ & \\
\hline
\end{tabular}

Fuente: elaboración propia.

9 En FAMILITUR existe una categoría de nivel de estudios denominada «Certificado escolar, EGB $1^{\circ}, 10$ años», que no se ha incluido en TURVERCON. Como esta categoría se encuentra entre «Sin Estudios o primarios sin terminar» $\mathrm{y}$ «Primarios terminados», para comparar ambas muestras se han agrupado los niveles de estudios desde «Primarios sin terminar» hasta «Primarios terminados». 
Tabla 5

DURACIÓN DE LA ESTANCIA

(PORCENTAJES SOBRE VIAJES DE MAYORES DE 15 AÑOS)

\begin{tabular}{lcccc}
\hline & \multicolumn{2}{c}{ TURVERCON } & \multicolumn{2}{c}{ FAMILITUR } \\
\hline & Viajes & Error & Viajes ${ }^{10}$ & Error \\
\hline $\begin{array}{l}\text { Entre una semana } \\
\text { y 15 días }\end{array}$ & 45,2 & 2,3 & 45,4 & 6 \\
\hline 16 días o más & 54,8 & 2,3 & 54,6 & 6 \\
\hline Total & $100 \%$ & & $100 \%$ & \\
\hline
\end{tabular}

Fuente: elaboración propia.

\section{Tabla 6 \\ SITUACIÓN LABORAL DEL ENTREVISTADO (PORCENTAJES SOBRE VIAJES DE MAYORES DE 15 AÑOS ${ }^{11}$}

\begin{tabular}{lcccc}
\hline & \multicolumn{2}{c}{ TURVERCON } & \multicolumn{2}{c}{ FAMILITUR } \\
\hline & Viajes & Error & Viajes & Error \\
\hline Estudiante & 8,4 & 1,3 & 11,5 & 3,8 \\
\hline $\begin{array}{l}\text { Jubilado o } \\
\text { pensionista }\end{array}$ & 22,1 & 1,9 & 16,8 & 4,5 \\
\hline $\begin{array}{l}\text { Ama de casa Tareas } \\
\text { del hogar }\end{array}$ & 12,8 & 1,5 & 13,9 & 4,2 \\
\hline
\end{tabular}

Fuente: elaboración propia.

10 Como en la submuestra de FAMILITUR VRCB6, hay algunos individuos mayores de 15 años que han realizado 2, e incluso 3, viajes de VRCB6 en 2008. La duración de la estancia en FAMILITUR se ha calculado sumando la duración de cada uno de los viajes, para poder comparar dicha duración con la obtenida a partir de TURVERCON, donde se preguntaba al entrevistado por el tiempo total que iba a permanecer de vacaciones en el destino.

11 Solo se muestran las categorías que son comparables entre ambas encuestas. 
